\title{
Transcript mapping of Cotton leaf curl Burewala virus and its cognate betasatellite, Cotton leaf curl Multan betasatellite
}

\author{
Fazal Akbar ${ }^{1,2}$, Rob W Briddon ${ }^{1}$, Franck Vazquez ${ }^{2}$ and Muhammad Saeed ${ }^{1 *}$
}

\begin{abstract}
Background: Whitefly-transmitted geminiviruses (family Geminiviridae, genus Begomovirus) are major limiting factors for the production of numerous dicotyledonous crops throughout the warmer regions of the world. In the Old World a small number of begomoviruses have genomes consisting of two components whereas the majority have single-component genomes. Most of the monopartite begomoviruses associate with satellite DNA molecules, the most important of which are the betasatellites. Cotton leaf curl disease (CLCUD) is one of the major problems for cotton production on the Indian sub-continent. Across Pakistan, CLCUD is currently associated with a single begomovirus (Cotton leaf curl Burewala virus [CLCUBuV]) and the cotton-specific betasatellite Cotton leaf curl Multan betasatellite (CLCUMuB), both of which have recombinant origins. Surprisingly, CLCuBuV lacks C2, one of the genes present in all previously characterized begomoviruses. Virus-specific transcripts have only been mapped for few begomoviruses, including one monopartite begomovirus that does not associate with betasatellites. Similarly, the transcripts of only two betasatellites have been mapped so far. The study described has investigated whether the recombination/mutation events involved in the evolution of CLCUBUV and its associated CLCUMuB have affected their transcription strategies.
\end{abstract}

Results: The major transcripts of CLCUBuV and its associated betasatellite (CLCUMuB) from infected Nicotiana benthamiana plants have been determined. Two complementary-sense transcripts of $\sim 1.7$ and $\sim 0.7 \mathrm{~kb}$ were identified for CLCUBuV. The $\sim 1.7 \mathrm{~kb}$ transcript appears similar in position and size to that of several begomoviruses and likely directs the translation of $\mathrm{C} 1$ and $\mathrm{C} 4$ proteins. Both complementary-sense transcripts can potentially direct the translation of $\mathrm{C} 2$ and $\mathrm{C} 3$ proteins. A single virion-sense transcript of $\sim 1 \mathrm{~kb}$, suitable for translation of the $V 1$ and V2 genes was identified. A predominant complementary-sense transcript was also confirmed for the betasatellite.

Conclusions: Overall, the transcription of CLCUBUV and the recombinant CLCUMuB is equivalent to earlier mapped begomoviruses/betasatellites. The recombination events that featured in the origins of these components had no detectable effects on transcription. The transcripts spanning the mutated C2 gene showed no evidence for involvement of splicing in restoring the ability to express intact C2 protein.

Keywords: Begomovirus, Cotton leaf curl disease, Cotton leaf curl Burewala virus, Betasatellite, Cotton leaf curl Multan betasatellite, Transcription

\footnotetext{
*Correspondence: saeed_hafeez@yahoo.com

${ }^{1}$ Agricultural Biotechnology Division, National Institute for Biotechnology and

Genetic Engineering, Jhang Road, Faisalabad, Pakistan

Full list of author information is available at the end of the article
} 


\section{Background}

Viruses of the Geminiviridae family have circular singlestranded (ss)DNA genomes and classify into four genera according to their host range, insect vector and genome organization [1]. They are widely distributed throughout the world and infect either monocotyledonous or dicotyledonous hosts. Geminiviruses of the genus Begomovirus are transmitted by the whitefly Bemisia tabaci and have genomes that consist of either a single or two ssDNA components. The two components of bipartite begomoviruses are known as DNA A and DNA B and both are, for most species, essential for symptomatic infection of plants. Monopartite begomoviruses are often associated with DNA satellites known as alphasatellites and betasatellites [2]. The satellite-associated begomoviruses are widespread in the Old World and represent the largest group of begomoviruses, outnumbering both the truly monopartite and the bipartite begomoviruses.

Geminiviruses replicate via a double-stranded (ds) DNA intermediate that is also used as a template for transcription. Transcription is bidirectional to generate mRNAs diverging from an intergenic region. Transcripts initiate downstream of either consensus TATA box motifs or initiator elements, suggesting that host RNA polymerase II transcribes the viral mRNAs. The viral RNAs are polyadenylated and composed of multiple RNA species, indicating the complexity of geminiviral transcription [3].

The genomes of monopartite begomoviruses encode six genes, two in the virion-sense orientation ( $V 1$ and $V 2$ ) and four in the complementary-sense orientation (C1 to $C 4)$. The $V 1$ gene encodes the coat protein (CP), involved in virus movement within and between plants [4,5], and the V2 protein which is involved in virus movement in plants as well as overcoming RNA silencing host defences triggered by dsRNA (also known as post-transcriptional gene silencing [PTGS]) for some virus species [6,7]. In the complementary-sense the $C 1$ gene encodes a rolling-circle replication initiator protein (known as the replication-associated protein [Rep]), that also interferes with host cell-cycle and is the only virus encoded protein required for virus replication, the $\mathrm{C} 2$ protein (known as the transcriptional-activator protein for some begomoviruses) that is involved in upregulating late, virion-sense encoded genes (in some cases) as well as host genes and is a RNA silencing suppressor [6,8-10]. The product of the $C 3$ gene, known as the replication enhancer protein (REn), is involved in virus DNA replication (by interacting with Rep) and also interacts with host components [11]. The product of the C4 gene may be a symptom determinant and also exhibits RNA silencing suppressor activity [6].

The betasatellites are a recently identified class of ssDNA satellites [2]. They are, in many cases, required by their helper begomoviruses to symptomatically infect the hosts from which they were isolated [12,13]. Betasatellites encode a dominant symptom/pathogenicity determinant (known as $\beta \mathrm{C} 1$ ) which is a suppressor of PTGS and may be involved in virus movement in plants and enhance virus DNA levels in plants [6,14-17].

Cotton leaf curl disease (CLCuD) is the most significant problem for cotton production across most of Pakistan and northwestern areas of India [18]. This disease appeared in epidemic form in 1991-92 and is caused by monopartite begomoviruses (seven distinct species were identified), often as multiple infections [19], and a specific betasatellite - Cotton leaf curl Multan betasatellite (CLCuMuB)[13]. In the late 1990's the introduction of resistant cotton varieties restored cotton production in Pakistan to pre-epidemic levels. However, from 20012002 onwards, the disease appeared on all previously resistant varieties, an indication of the appearance of a strain of the disease with the ability to break resistance (now known as the "Burewala" strain) [20]. CLCuD in resistant cotton varieties has been shown to be associated with a single begomovirus, Cotton leaf curl Burewala virus $(\mathrm{CLCuBuV})$, which has spread across most areas of Pakistan and into India [21-23]. CLCuBuV is a recombinant virus consisting of sequences derived from two parents, Cotton leaf curl Kokhran virus (CLCuKoV; which donated the virion-sense sequences) and Cotton leaf curl Multan virus (CLCuMuV; which donated the complementary-sense sequences). These two species were dominant in cotton prior to resistance breaking $[19,21]$. Significantly, CLCuBuV lacks an intact $C 2$ gene. This is surprising since all begomoviruses, curtoviruses and the only known topocurvirus reported to date have a $C 2$ gene that potentially encodes an $~ 134$ aa protein [21]. C2 is a multifunctional protein that plays an important role in host-virus interactions.

The betasatellite associated with $\mathrm{CLCuBuV}$ was also shown to be recombinant. This consists of the original $\mathrm{CLCuMuB}$ with a small fragment ( 80 nt), in a noncoding sequence, derived from a betasatellite first identified in tomato [21,24]. In common with all betasatellites, $\mathrm{CLCuMuB}$ encodes a single gene, $\beta C 1$.

The work presented here consisted of mapping the major transrcripts of $\mathrm{CLCuBuV}$ and its associated CLCu$\mathrm{MuB}$ for comparison to the transcript maps of other begomoviruses/betasatellites and investigating whether the recombination/mutation events involved in their evolution have affected gene expression at the level of transcription.

\section{Methods}

\section{Infection of plants and RNA isolation}

The begomovirus clones CLCuBuV-[PK:Veh2:4] (accession number AM421522) and associated betasatellite, CLCuMuB-[PK:Veh:06] (AM774307) were used to infect $N$. benthamiana plants as previously described [21]. 
Total RNA was isolated from plants using Trizol reagent (Gibco-BRL) as described by the manufacturer. The extracted RNA was dissolved in diethylpyrocarbonatetreated water and stored at $-80^{\circ} \mathrm{C}$.

\section{Northern blot hybridization}

Total RNA $(10 \mu \mathrm{g})$ was electrophoresed on $1.2 \%$ agarose MOPS gels, blotted onto nylon membranes (Hybond $\mathrm{N}$ +; Amersham) and UV cross-linked. DNA fragments for virus complementary-sense (coordinates 1059-66) and virion-sense (coordinates 124-1059) probes were PCR amplified using specific primers CF/CR and VF/VR (Table 1), respectively, and labeled with $\left[\alpha-{ }^{32} \mathrm{P}\right] \mathrm{dCTP}$ using a Megaprime labeling kit (Amersham). Hybridization was performed at $45^{\circ} \mathrm{C}$ for $16 \mathrm{~h}$. Following stringent washing, radioactive signals were detected using a storage phosphor screen and the images were acquired after $3 \mathrm{~h}$ exposure using a Typhoon phosphoimager (Amersham). A betasatellite specific DNA probe was derived from the complete coding region of the $\beta C 1$ gene, labelled with a DIG PCR labelling kit and exposed to Xray film after treatment with CDP-Star (Roche).

\section{5 ' RNA ligase-mediated rapid amplification of cDNA ends (RLM-RACE) PCR}

$5^{\prime}$ RLM-RACE PCR was performed using an RLMRACE Kit (Ambion) according to the manufacturer's instructions. Total RNA was treated with alkaline phosphatase to remove the $5^{\prime}$ phosphate group of noncapped mRNAs, followed by treatment with tobacco acid pyrophosphatase (TAP) to decap mRNAs. TAP treated RNA was ligated to a synthetic $5^{\prime}$ RNA adapter (Table 1) using T4 RNA Ligase. This RNA was reverse transcribed and PCR was performed with specific primer combinations (Table 1 ) to amplify $5^{\prime}$ termini of virus and betasatellite specific transcripts.

\section{3' RLM-RACE PCR}

$3^{\prime}$ RLM-RACE PCR was performed according to the manufacturer's instructions (Ambion). Total RNA $(1 \mu \mathrm{g})$ was reverse transcribed with an oligo (dT) adapter

Table 1 Oligonucleotide primers used in the study

\begin{tabular}{|c|c|c|}
\hline Primer & Oligonucleotide sequence $\left(5^{\prime}\right.$ to $\left.3^{\prime}\right)$ & Nucleotide coordinates* \\
\hline BuC2R & TCATTCAAGATCTACTCTCC & $1500-1520$ \\
\hline $\mathrm{REn}^{\prime}{ }^{\prime} 5 \mathrm{~B}$ & GCTGTGAGGTCATCCAGATTC & $1235-1256$ \\
\hline $\mathrm{V} 2 \mathrm{CP}-5 \mathrm{~B}$ & TAGGAACATCTGGACTTCTGT & 488-466 \\
\hline V2cpB2 & ACCGTGAACGGTGTCGGGGA & $176-156$ \\
\hline $\mathrm{BC} 2$ & TGCTCCCTTCAAAGCCGT & $358-376$ \\
\hline $\mathrm{BC} 2 \mathrm{bb}$ & GAGATCGAGATAGAAGATATAG & $314-292$ \\
\hline $\mathrm{BC} 1-{ }^{\prime} 3$ & CAAGTATGAAGGGATCGTCCA & $435-415$ \\
\hline $\mathrm{BC} 1-{ }^{\prime} 5$ & TGGACGATCCCTTCATACTTG & $415-435$ \\
\hline $\mathrm{BuC2F}$ & ATGCAACCTTCATCACTCTC & $1608-1587$ \\
\hline REn-'3B & ATGGATTCACGCACAGGGGA & $1463-1443$ \\
\hline $5^{\prime}$ RACE outer & GCTGATGGCGATGAATGAACACTG & - \\
\hline 5'RACE inner & CGCGGATCCGAACACTGCGTITGCTGGCTTGATG & - \\
\hline 3'RACE outer & GCGAGCACAGAATTAATACGACT & - \\
\hline 3'RACE inner & CGCGGATCCGAATTAATACGACTCACTATAGG & - \\
\hline $\mathrm{C} 1 \mathrm{C} 4-^{\prime} 5 \mathrm{~K}$ & CTAGTTCCTTAATGACTC & $2137-2155$ \\
\hline $\mathrm{C} 1 \mathrm{C} 4-5^{\prime} \mathrm{K} 2$ & AACGTTGGGGGGAGCCAT & $2577-2596$ \\
\hline $\mathrm{C} 1 \mathrm{C} 4 \mathrm{~B} 1$ & ATGGCTCCCCCCAAACGTT & $2596-2577$ \\
\hline v2cpb1 & ATGTCGAAGCGACCAGCAGATATAATC & $292-318$ \\
\hline $\mathrm{c} 1 \mathrm{c} 4 \mathrm{~b} 2$ & ATTGTCTCCAAATGGCAT & 2664-2682 \\
\hline v2cpb2b & GCGTACCTTCGAAGCGGGCGTGGAAAT & $320-346$ \\
\hline$v 2 c p-3 b$ & ACAGAAGTCCAGATGTTCCTAG & $466-488$ \\
\hline VF & CTTCGTTGCTAAGTTTGCG & $105-123$ \\
\hline VR & ATTGTCACGGAATCATAGA & 1059-1039 \\
\hline CF & GCGTCATATGATTGGCCGAC & $66-46$ \\
\hline CR & TATTGAAGATGATTGGTCTA & 1076-1096 \\
\hline
\end{tabular}

*CLCuBuV nucleotide coordinates are according to acc. no. AM421522 [21]. CLCUMuB nucleotide coordinates are according to acc. no. AM774307 [21]. 
primer (Table 1), and cDNA was used in PCR with specific primer combinations (Table 1 ) to amplify $3^{\prime}$ termini of virus and betasatellites specific transcripts.

\section{Cloning and sequencing of RLM-RACE PCR products}

PCR products were purified using a gel extraction kit (Fermentas) according to the manufacturer's instructions, and were cloned into the $\mathrm{pTZ} 57 \mathrm{R} / \mathrm{T}$ vector (Fermentas). PCR products (without cloning), as well as cloned products, were sequenced (Macrogen, Korea). Sequences were analyzed using the Lasergene software package (DNASTAR Inc.).

\section{Results}

Northern blot analysis of virus-specific RNAs in CLCuBuV/ CLCuMuB-infected $N$. benthamiana plants

RNA gel blots of total RNA extracted from virusinfected $N$. benthamiana plants are shown in Figure 1. Hybridization with the $\mathrm{CLCuBuV}$ complementary-sense probe identified two major specific RNAs of $\sim 1.7$ and $\sim 0.7 \mathrm{~kb}$ in $\mathrm{CLCuBuV} / \mathrm{CLCuMuB}$-infected plant tissue (Figure 1, panel A). Hybridization with the virion-sense probe revealed a single predominant RNA of $\sim 1 \mathrm{~kb}$ in infected plants (Figure 1, panel B). No hybridization was detected in extracts from healthy, non-inoculated $N$. benthamiana tissue to either probe. In addition to the single long transcript on the blot probed with the virionsense probe, a smear was observed at the bottom of the blots. This most likely represents degradation products of the major transcript (Figure 1, panel B). Two additional hybridization signals for virion-sense (of approximately 300 and 500nt) are shown by asterisks. The blots were repeated twice with independent samples and the same results were obtained each time.

\section{High-resolution mapping of the CLCuBuV complementary-sense transcripts}

The location of $5^{\prime}$ ends of complementary-sense transcripts were determined by $5^{\prime}$ RLM-RACE PCR. Sequencing of clones generated by RLM-RACE, using the primer pair REn $-5^{\prime} \mathrm{B}$ and $5^{\prime}$ RACE outer primer and then nested PCR using primer pair $\mathrm{BuC} 2 \mathrm{R}$ and $5^{\prime} \mathrm{RACE}$ inner primer (Table 1), indicated ligation of the RNA adapter primer to RNA with $5^{\prime}$ ends at positions 1628, 1636 and 1752. This transcript mapped upstream of the $C 2$ and $C 3$ and thus most likely represents the $5^{\prime}$ ends for the small $\mathrm{CLCuBuV}$ transcript of $\sim 0.7-\mathrm{kb}$ (Figure 2). Four other transcripts were identified, the $5^{\prime}$ ends of which mapped at positions 2632, 2709, 2721 and 66 using gene specific primer $\mathrm{C} 1 \mathrm{C} 4-5^{\prime} \mathrm{K}$ and $5^{\prime}$ RACE outer primer and then using inner primer C1C4-5' K2 or c1c4b2 with 5/RACE inner primer (Table 1) in a nested PCR. The transcript mapped at position 2632 initiates upstream of the $C 1$ gene, while the longer transcript mapped at positions 2709, 2721 and 66 lies upstream of the $C 4$ gene (Figure 3 ).

The locations of $3^{\prime}$ ends for complementary-sense transcripts were examined by $3^{\prime}$ RLM-RACE PCR. The gene specific primers $\mathrm{C} 1 \mathrm{C} 4 \mathrm{~B} 1$ (outer) and $\mathrm{BuC} 2 \mathrm{~F}$ (inner) were used in combination with $5^{\prime}$ RACE outer and inner primers respectively for the $3^{\prime}$ end of $\sim 1.7 \mathrm{~kb}$ transcripts. Similarly the outer primer $\mathrm{BuC} 2 \mathrm{~F}$ and inner primer REn-'3B were used for the $3^{\prime}$ end of small transcript of $\sim 0.7 \mathrm{~kb}$. Sequencing results showed that all the complementary-sense transcripts have a single major transcription termination site mapping at coordinate 1059 (Figure 4).

\section{High-resolution mapping of the CLCuBuV virion-sense transcripts}

The gene specific primer V2CP-'5B and the 5 'RACE outer primer were used in the primary PCR and then primer v2cpb2b was used with the $5^{\prime} \mathrm{RACE}$ inner primer in a nested PCR to determine the $5^{\prime}$ ends of virion-sense transcripts. Sequence analysis revealed the presence of two predominant $5^{\prime}$ ends, at nucleotides 106 and 147, for the virion-sense transcripts of $\mathrm{CLCuBuV}$ (Figure 5), which are consistent with the $\sim 1 \mathrm{~kb}$ transcript determined by northern blotting (Figure 1). In addition a

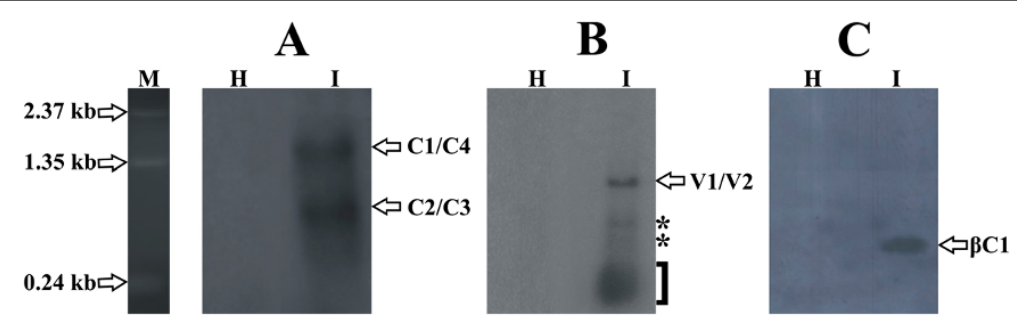

Figure 1 Northern blot analysis of the transcripts of CLCuBuV. Northern blot of total RNA extracted from a healthy Nicotiana benthamiana plant $(\mathrm{H})$ and a plant infected with CLCUBuV/CLCUMUB (I). Detection of complementary- and virion-sense specific RNAs in infected plants are shown in panels A and B respectively. Immobilized RNA was hybridized to complementary- $(\mathbf{A})$ and virion-sense probes (B). Detection of CLCuMuB specific RNA is shown in panel C. Approximately equal amounts $(10 \mu \mathrm{g})$ of RNA were loaded in each well. Estimated sizes are given in kilobases (kb) based on the positions of a co-electrophoresed RNA marker (M). Two additional hybridization signals (indicated by the asterisks) and the smear at the bottom of the virion-sense probed blot (indicated by the bracket on the right) is discussed in the text. 
1397

CTT CCC AGA TAT ATG CGC CAT TCC CTG CTT GAG CTG CAG TGA TGG GTT CCC CTG TGC GTG GAA GGG TCT ATA TAC GCG GTA AGG GAC GAA CTC GAC GTC ACT ACC CAA GGG GAC ACG CAC

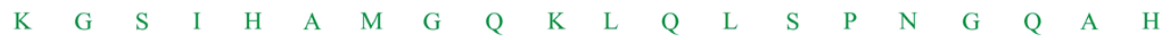
$\begin{array}{llllllllllllllllllll}\text { E } & \text { W } & \text { I } & \text { Y } & \text { A } & \text { G } & \text { N } & \text { G } & \text { A } & \text { Q } & \text { A } & \text { A } & \text { T } & \text { I } & \text { P } & \text { E } & \text { G } & \text { T } & \text { R } & \text { S }\end{array}$

1457

AAT CCA TGG TTG TGG CAG TTG ATT GAC AGA TAC TAA GAA CAC CCT CAT TCA AGA TCT ACT TTA GGT ACC AAC ACC GTC AAC TAA CTG TCA ATG ATT CTT GTG GGA GTA AGT TCT AGA TGA

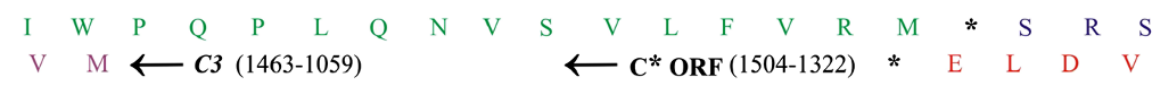

1517

CTC CTC CTC CTG TTG CGT CTC TTC GCT TCC CTG TGC TGT ACT TTG ATT GGA AGC TGA GTA GAG GAG GAG GAC AAC GCA GAG AAG CGA AGG GAC ACG ACA TGA AAC TAA CCT TCG ACT CAT

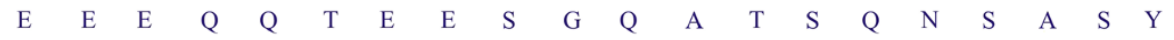
$\begin{array}{llllllllllllllllllllll}\mathrm{R} & \mathrm{R} & \mathrm{R} & \mathrm{R} & \mathrm{N} & \mathrm{R} & \mathrm{R} & \mathrm{K} & \mathrm{A} & \mathrm{E} & \mathrm{R} & \mathrm{H} & \mathrm{Q} & \mathrm{V} & \mathrm{K} & \mathrm{I} & \mathrm{P} & \mathrm{L} & \mathrm{Q} & \mathrm{T}\end{array}$

1577

CAG TGG TCC TTC GAG AGT GAT GAA GGT TGC ATT TTT TAA GGC CCA ATT CTT TAA TGA TGT GAC ACC AGG AAG CAC TCA CTA CTT CCA ACG TAA AAA ATT CCG GGT TAA GAA ATT ACT ACA

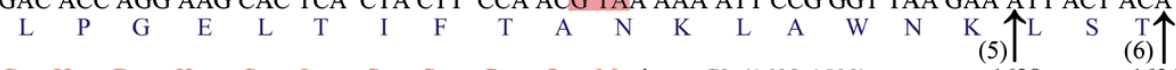

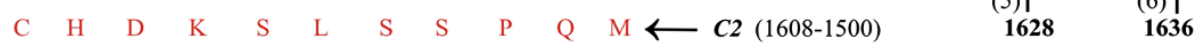

1637

GTT TTT TCC CTC GTT GAG GAA TTC ATT ATA ACT GCT GTT CGG ACC AGG ATT GCA CAG GAA CAA AAA AGG GAG CAA CTC CTT AAG TAA TAT TGA CGA CAA GCC TGG TCC TAA CGT GTC CTT $\begin{array}{llllllllllllllllllllll}\mathrm{N} & \mathrm{K} & \mathrm{G} & \mathrm{E} & \mathrm{N} & \mathrm{L} & \mathrm{F} & \mathrm{E} & \mathrm{N} & \mathrm{Y} & \mathrm{S} & \mathrm{S} & \mathrm{N} & \mathrm{P} & \mathrm{G} & \mathrm{P} & \mathrm{N} & \mathrm{C} & \mathrm{L} & \mathrm{F}\end{array}$ $\leftarrow C 1(2596-1505)$

1697 GAT TGT CGG TAT CCC GCC TTT AAT TTG AAC TGG CTT TCC GTA TTT TGT GTT GGA CTG CCA CTA ACA GCC ATA GGG CGG AAA TTA AAC TTG ACC GAA AGG CAT AAA ACA CAA CCT GAC GGT

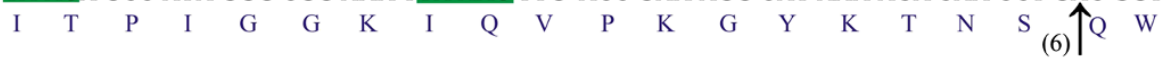
1752

1757 GTC TCT TTG GGC CCC CAT GAA CTC TTT AAA GTG TTT GAG GAA ATG CGG GTC GAC GTC ATC CAG AGA AAC CCG GGG GTA CTT GAG AAA TTT CAC AAA CTC CTT TAC GCC CAG CTG CAG TAG $\begin{array}{llllllllllllllllllllll}\mathrm{D} & \mathrm{R} & \mathrm{Q} & \mathrm{A} & \mathrm{G} & \mathrm{M} & \mathrm{F} & \mathrm{E} & \mathrm{K} & \mathrm{F} & \mathrm{H} & \mathrm{K} & \mathrm{L} & \mathrm{F} & \mathrm{H} & \mathrm{P} & \mathrm{D} & \mathrm{V} & \mathrm{D} & \mathrm{D}\end{array}$

1817 AAT GAC GTT GTA CCA GGC GTC GTT ACT GTA TAC TTT GGG ACT CAG GTC CAG ATG TCC GCA TTA CTG CAA CAT GGT CCG CAG CAA TGA CAT ATG AAA CCC TGA GTC CAG GTC TAC AGG CGT $\begin{array}{lllllllllllllllllllll}\text { I } & \text { V } & \text { N } & \text { Y } & \text { W } & \text { A } & \text { N } & \text { D } & \text { S } & \text { Y } & \text { V } & \text { K } & \text { P } & \text { S } & \text { L } & \text { D } & \text { L } & \text { H } & \text { G } & \text { C }\end{array}$

Figure 2 Mapping the $5^{\prime}$ ends of the short CLCuBuV C2 and C3 gene transcripts. The nucleotide sequence of CLCuBuV between coordinates 1397-1876 is shown. The beginning of genes C2 and C3 are indicated by the start codon (ATG; highlighted in pink) and with the translations shown in red and purple text. The $\mathrm{C}$-terminal end of the $\mathrm{Cl}$ gene translation is shown in blue. The translation of a pseudo-gene $\left(\mathrm{C}^{*}\right)$, resulting from the frame-shift mutation of the $C 2$ gene (in frame with the $C 1$ gene) is shown in green text with the start codon highlighted with an orange box. The putative promoter TATA elements and CAAT boxes are highlighted with purple and green boxes, respectively. The position of independent RACE clone ends mapping to the $5^{\prime}$ termini are indicated by their coordinates and with arrows. The numbers of cDNA clones sequenced for each end are indicated in brackets. Nucleotide numbering is according to [21].

minor virion-sense transcript, mapping at nucleotide 180, was detected.

The $3^{\prime}$ RACE outer primer was used in combination with the gene specific primer $\mathrm{v} 2 \mathrm{cpb} 1$ in the primary PCR and then nested PCR was performed using the $3^{\prime}$ RACE inner primer and gene specific primer v2cp-'3b to determine the $3^{\prime}$ ends of virion-sense transcripts. The transcripts spanning genes $V 1$ and $V 2$ had the same predominant $3^{\prime}$ end with the transcription termination site mapping at nt 1083 and $3^{\prime}$ untranslated regions (UTRs) of 21nt (Figure 4).

Mapping the $3^{\prime}$ and $5^{\prime}$ ends of $\beta C 1$ transcript of CLCuMuB Sequence analysis of $5^{\prime}$ RLM-RACE clones for CLCu$\mathrm{MuB}$ revealed the presence of one predominant $5^{\prime}$ end. The primer pair $\mathrm{BC} 2$ and $5^{\prime} \mathrm{RACE}$ outer primer were 


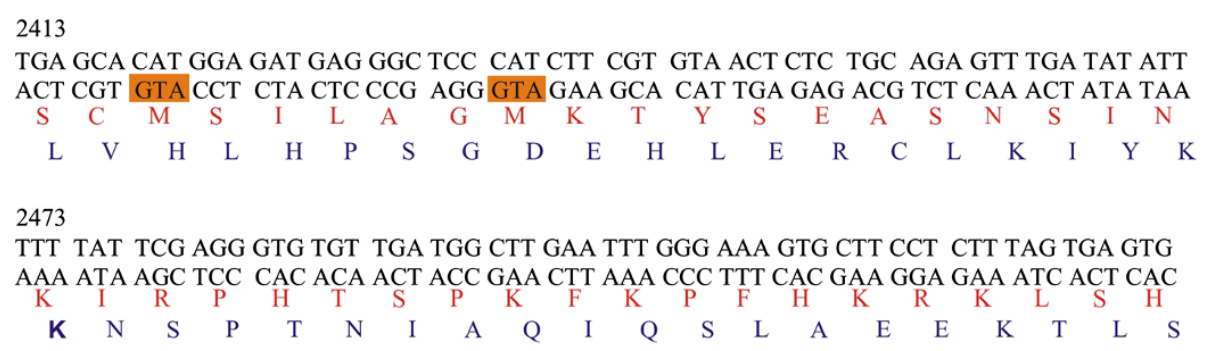

Figure 3 Mapping the $5^{\prime}$ ends of CLCuBuV C1 and C4 gene transcripts. The nucleotide sequence of CLCuBuV between coordinates $2413-$ 133 is shown. The beginning of genes $\mathrm{Cl}$ and $\mathrm{C} 4$ are indicated by the start codon (ATG; highlighted in pink). The predicted amino acid sequence of the $\mathrm{C} 1$ and $\mathrm{C} 4$ proteins are shown in blue and red text, respectively. The putative promoter TATA elements and CAAT boxes are highlighted with purple and green boxes, respectively. The position of independent RACE clone ends mapping to the $5^{\prime}$ termini are indicated with arrows and by their coordinates. The numbers of cDNA clones sequenced for each end are indicated in brackets. The conserved, between geminiviruses, nonanucleotide sequence (TAATATTAC) is highlighted with a large pink box. The two internal methionine codons (highlighted in orange) of the C4 gene are discussed in the text. Nucleotide numbering is according to [21].

used in primary PCR and then nested PCR was performed using primer pair BC1-' 5 and 5 ' RACE inner primer (Table 1), indicated ligation of the RNA adapter primer to RNA with a $5^{\prime}$ end at position 563 (Figure 6).

$3^{\prime}$ RLM-RACE clones showed transcription termination mapping at coordinate 190 (Figure 6). Gene specific primers $\mathrm{BC} 1-3^{\prime}$ (outer) and $\mathrm{BC} 2 \mathrm{bb}$ (inner) were used in combination with $3^{\prime}$ RACE outer and inner primers respectively (Table 1 ).

\section{Discussion}

Although the transcription strategies of a few begomoviruses have been determined [25-29], including one monopartite begomovirus (Tomato leaf curl virus [ToLCV])[28], none of the betasatellite-associated begomoviruses are amongst these. Only for two betasatellites, Ageratum yellow vein betasatellite (AYVB) and the nonrecombinant $\mathrm{CLCuMuB}[15,30]$, have transcripts been determined. It was therefore of interest to determine the 


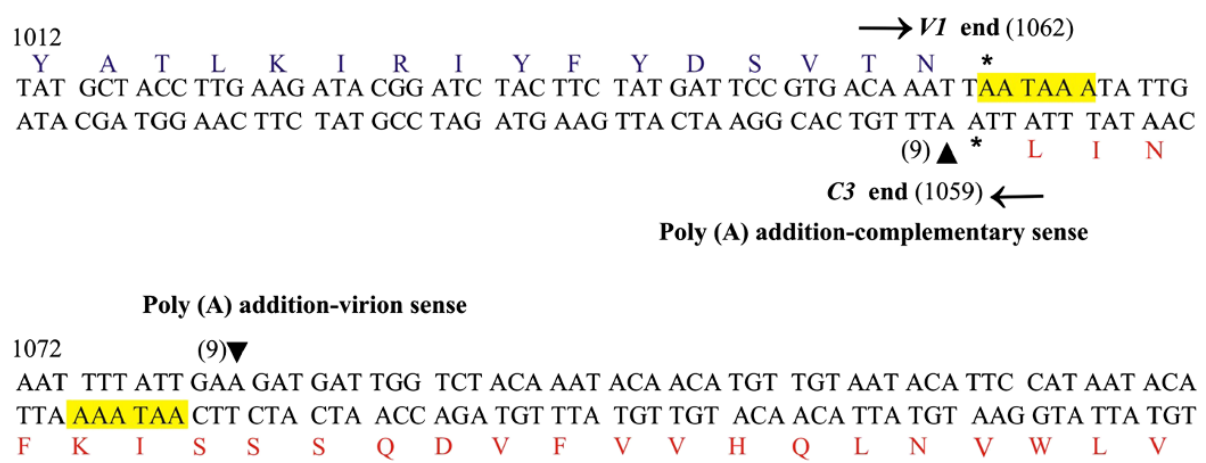

Figure 4 Mapping the $\mathbf{3}^{\prime}$ ends of complementary- and virion-sense transcripts of CLCuBuV. The nucleotide sequence of CLCuBuV between coordinates 1012-1131 is shown. The $3^{\prime}$ ends for transcripts are indicated by black triangles. The numbers of cDNA clones sequenced for each end are indicated in brackets. The translation stop codons of the $V 1$ and $C 3$ genes are indicated with asterisks. Polyadenylation signals are highlighted in yellow. Nucleotide numbering is according to [21].

transcripts of $\mathrm{CLCuBuV}$, and its associated recombinant betasatellite. Particular attention was paid to transcription across the mutation affecting the CLCuBuV C2 gene to determine whether any transcriptional changes resulted from the mutation.
In common with other geminiviruses $\mathrm{CLCuBuV}$ produces multiple overlapping polycistronic RNA species that diverge from the IR, confirming a bidirectional transcription strategy. Transcription of the complementary-sense is more complex, producing multiple RNAs species with

1 ACC GGA TGG CCG CGC GAT TTT TTC GTG GGC CCT ACC ATT AAC TCT TGT CGG CCA ATC AT TGG CCT ACC GGC GCG CTA AAA AAG CAC CCG GGA TGG TAA TTG AGA ACA GCC GGT TAG TA 60
ATG ACG CGC TCA AAG CTT AAA TAA TTC TCC CGC CTA TTA TAA GTA CTT CGT TGC TAA GTT
TAC TGC GCG AGT TTC GAA TTT ATT AAG AGG GCG GAT AAT ATT CAT GAA GCA ACG ATT CAA

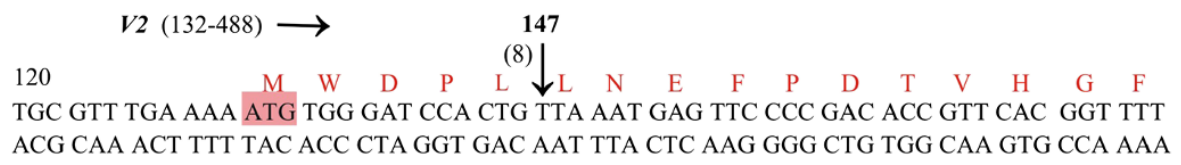

180

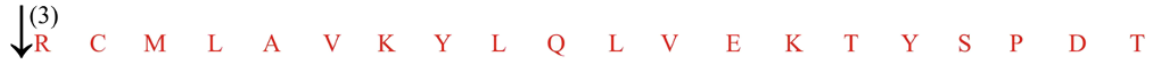
AGG TGT ATG TTA GCA GTT AAA TAT TTG CAG TTA GTA GAG AAA ACT TAC TCT CCT GAT ACA TCC ACA TAC AAT CGT CAA TTT ATA AAC GTC AAT CAT CTC TTT TGA ATG AGA GGA CTA TGT

$$
\begin{aligned}
& 240 \quad \text { V1 (292-1062) } \rightarrow \text { M S K }
\end{aligned}
$$

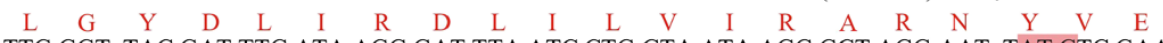

$$
\begin{aligned}
& \text { TTG GGT TAC GAT TTG ATA AGG GAT TTA ATC CTG GTA ATA AGG GCT AGG AAT TAT GTC GAA } \\
& \text { AAC CCA ATG CTA AAC TAT TCC CTA AAT TAG GAC CAT TAT TCC CGA TCC TTA ATA CAG CTT } \\
& 300 \\
& \begin{array}{llllllllllllllllllllllllllll}
R & P & A & D & I & I & I & S & T & P & A & S & K & V & R & R & R & L & N & F
\end{array} \\
& \begin{array}{lllllllllllllllllllll}
A & T & S & R & Y & N & H & F & H & A & R & F & E & G & T & P & P & S & Q & L
\end{array} \\
& \text { GCG ACC AGC AGA TAT AAT CAT TTC CAC GCC CGC TTC GAA GGT ACG CCG CCG TCT CAA CTT } \\
& \text { CGC TGG TCG TCT ATA TTA GTA AAG GTG CGG GCG AAG CTT CCA TGC GGC GGC AGA GTT GAA }
\end{aligned}
$$

Figure 5 Mapping the $\mathbf{5}^{\prime}$ ends of CLCuBuV $\mathbf{V} \mathbf{1}$ and $\mathbf{V} \mathbf{2}$ gene transcripts. The nucleotide sequence of CLCuBuV between coordinates 1-360 is shown. The beginning of genes $V 2$ and $V 1$ are indicated by the start codon (ATG; highlighted in pink). The predicted amino acid sequence of the V1 and V2 proteins are shown in red and blue text, respectively. The putative promoter TATA elements and CAAT boxes are highlighted with purple and green boxes, respectively. The position of independent RACE clone ends mapping to the $5^{\prime}$ termini are indicated by their coordinates and with arrows. The numbers of cDNA clones sequenced for each end are indicated in brackets. Nucleotide numbering is according to [21]. 
Figure 6 Mapping the $\beta C 1$ transcript of CLCuMuB. The nucleotide sequence of CLCUMuB between coordinates 181-240 and 540-599 is shown. The beginning of the $\beta C 1$ gene is indicated by the start codon (ATG; highlighted in pink) and the end by the stop codon (indicated with an asterisk). The numbers of cDNA clones sequenced for each end are indicated in brackets. The putative promoter TATA element is highlighted in purple and polyadenylation signal in yellow. The position of independent RACE clone ends mapping to the $5^{\prime}$ termini are indicated by arrows with coordinates and the $3^{\prime}$ termini by a black triangles. Nucleotide numbering is according to [21].

distinct $5^{\prime}$ ends and a common $3^{\prime}$ end. The polyadenylation sites are arranged such that complementary- and virion-sense RNAs overlap at their $3^{\prime}$ ends [29,31]. Though complementary and virion-sense transcripts overlap only by a small region, this unusual read-through transcription on a circular viral DNA could produce longer transcripts that are complementary, forming dsRNA, that may act to trigger RNA silencing [29,32]. Hybridization smears (Figure 1) detected on northern blots may represent the breakdown products of RNA formed from such aberrant read-through transcription. The additional hybridization signals detected on northern blots may represent short ORFs (Figure 1 panel, B). Such additional signals representing short ORFs have been mapped for the BC1 transcription unit of Mungbean yellow mosaic virus (MYMV)[29]. These short ORFs may inhibit the translation of downstream genes unless leaky scanning occurs. Leaky scanning occurs when the $5^{\prime}$ most initiation codon has a weak context and ribosomes instead initiate translation at a downstream codon [33].

The virion-sense transcripts of $\mathrm{CLCuBuV}$ are comparable in size and location to those of other begomoviruses and the curtovirus Spinach curly top virus (SpCTV) [28,31]. Mapping of the virion-sense transcripts of $\mathrm{CLCuBuV}$ identified two major $5^{\prime}$ termini. One $5^{\prime}$ end mapped upstream of the $V 1$ and $V 2$ genes and could potentially direct the translation of both of these genes, whereas the second $5^{\prime}$ end mapped upstream of $V 1$, and could potentially serve to translate only the CP (Figure 5). The transcript spanning the $V 1$, with $5^{\prime}$ end mapped at coordinate 147, has an untranslated leader of $145 \mathrm{nt}$. An untranslated leader of $160 \mathrm{nt}$ has previously been reported for the $V 1$ gene of ACMV [25]. Analysis of the sequences of virion-sense transcripts of $\mathrm{CLCuBuV}$ indicated the presence of TATA box sequences at 34 and 49 nt upstream of the $V 2$ start methionine codon. The size of RNAs characterised here is consistent with the $\sim 1 \mathrm{~kb}$ virion-sense RNA detected in infected tissue (Figure 1, panel B).
The analysis detected no RNAs that span only the C3, suggesting that REn is expressed only from polycistronic mRNAs. The transcripts spanning the $C 2$ and $C 3$ genes are of two classes, short (initiating at coordinates 1628 and 1636) and long (initiating at 1752)(Figure 2). The two transcripts have short $5^{\prime}$ UTRs of 20 and $28 \mathrm{nt}$, whereas the long transcript has a longer $5^{\prime}$ UTR of 144 nt (Figure 2). Tomato golden mosaic virus (TGMV) also transcribes to give two size classes of transcripts encompassing $C 2$ and $C 3$ [34]. For TGMV there are additional AUG initiation codons in the $5^{\prime}$ UTR of the longer C2/ C3 transcript. The first of these has the capacity to translate the C-terminal 122aa of the Rep, terminating after the $C 2$ initiation codon, was shown to be inhibitory for $\mathrm{C} 2$ and REn expression [34]. For CLCuBuV there is a single AUG initiation codon in the $5^{\prime}$ UTR of the 1752 transcript (Figure 2), out of frame with the $C 1$, which terminates 11 aa before the C2 AUG initiation codon. This is unlikely to significantly affect $C 2$ translation since the additional AUG initiation codon in the $5^{\prime}$ UTR is in an unfavourable context (tAtAAUGaaU) compared to the consensus context for dicot mRNAs (aaA[A/C] aAUGGCu)[35], whereas both the $C 2$ and $C 3$ AUG initiation codons are in a more favourable context (AAAAAAUGca and cAACcAUGGa). Thus in contrast to TGMV, CLCuBuV may translate both $\mathrm{C} 2$ and REn from both the size classes of $C 2 / C 3$ transcripts.

The mechanism by which REn is expressed is unclear. The gap between both the $C 1$ and (truncated) $C 2$ terminator codons and the start of $C 3$ suggests that it may involve reinitiation or internal initiation. For TGMV the translation of $\mathrm{C} 3$ from the short transcript may occur by leaky scanning, since the $C 3$ initiation codon occurs before the $\mathrm{C} 2$ terminator codon [34]. However, for $\mathrm{CLCu}-$ $\mathrm{BuV}$ the frame-shift mutation has resulted in the $\mathrm{C} 2$ termination codon occurring before the $C 3$ initiation codon and there is an ORF (indicated as $\mathrm{C}^{*}$ in Figure 2; this ORF would not encode $\mathrm{C} 2$ amino acid sequences), in-frame with $C 1$ and immediately after the $C 1$ 
termination codon), which could possibly be expressed by leaky scanning through the $C 2$ initiation codon. Translation of the C* ORF might adversely affect $C 3$ expression. However, further studies will be required to investigate these possibilities.

It remains unclear why geminiviruses would express REn from multiple transcripts. A possible explanation is that this protein may be required in larger amounts than possible from a single transcript or its expression requires more subtle control. The protein may be required at several stages of the virus infection cycle and thus may be expressed at different times from different mRNAs.

The transcript $5^{\prime}$ ends identified represent heterogeneous initiation sites of a bicistronic mRNA encoding both $C 2$ and $C 3$. The predicted sizes of RNAs initiating at the $5^{\prime}$ and $3^{\prime}$ ends mapped here is consistent with the complementary-sense RNAs detected in infected tissue. Identified transcription start sites are located downstream of putative TATA boxes at optimal distances of 20 to 35 nt. These results suggest that CLCuBuV uses a bicistronic transcription strategy, in common with other geminiviruses, to translate $\mathrm{C} 2$ and REn from a single transcript [29]. This is supported by the fact that $C 3$ gene-specific primers did not reveal any major transcription start between the $C 2$ and $C 3$ start codons, or splicing to remove the upstream $\mathrm{C} 2$ start codon.

The long $(\sim 1.7 \mathrm{~kb})$ complementary-sense transcripts could potentially allow translation of the $C 1$, truncated $C 2$ and $C 3$ genes (Figure 7). With the possible exception of the transcript initiating at coordinate 2632, all the long complementary-sense transcripts are also suitable for translation of C4. Transcript 2632 initiates downstream of the predicted AUG (coordinate 2682) of the C4 gene and would thus appear not to direct translation of the C4 (Figures 3). However, CLCuBuV clone used here is unusual in encoding a predicted 181aa C4; most begomoviruses have a much smaller $\mathrm{C} 4$ (although the size is variable, typically $\sim 85 \mathrm{aa}$ ). Also, a minority of $\mathrm{CLCuBuV}$ isolates encode a predicted $\mathrm{C} 4$ of $100 \mathrm{aa}$, although such clones have not so far been shown to be infectious to plants. The clone used here has two in-frame AUG codons (coordinates 2475 and 2493) within the C4 sequence which might initiate the translation of products of 100 and 94aa, respectively, more in-keeping with the normal size of begomovirus $\mathrm{C} 4$ proteins. It is thus possible that the predicted long $\mathrm{C} 4$ gene here is an artefact and that a more conventional $\mathrm{C} 4$ protein is translated from transcript 2632, or even that two distinct size classes of $\mathrm{C} 4$ protein are produced - 100aa from transcript 2632 and 181aa from the remaining transcripts. Which of these possibilities is correct will require further investigation.

For the long complementary-sense transcripts TATA and CAAT boxes are located at the requisite distances of approximately 30bp from the transcription initiation sites (Figure 3). The longer $1.7 \mathrm{~kb}$ transcript appears to use the invariant nonanucleotide sequence on the complementary strand as the promoter element (TATA box). Interestingly, the longest transcript maps at nt 66 with a $5^{\prime}$ UTR of $143 \mathrm{nt}$ that spans the origin of replication and promoter elements far from the complementary-sense genes. A similar transcript has previously been observed for TGMV [36]. For CLCuBuV the short $5^{\prime}$ UTRs are 20-40 nt long and the promoter elements are located at an optimal distance from transcription initiation sites as reported previously for others geminiviruses and thus represent the authentic $5^{\prime}$ ends for these transcripts [31]. For the longer $5^{\prime}$ UTRs, these elements are far removed from the transcription initiation sites. It has been recognized that translation initiation efficiency from the start codon (AUG) depends on its position and sequence

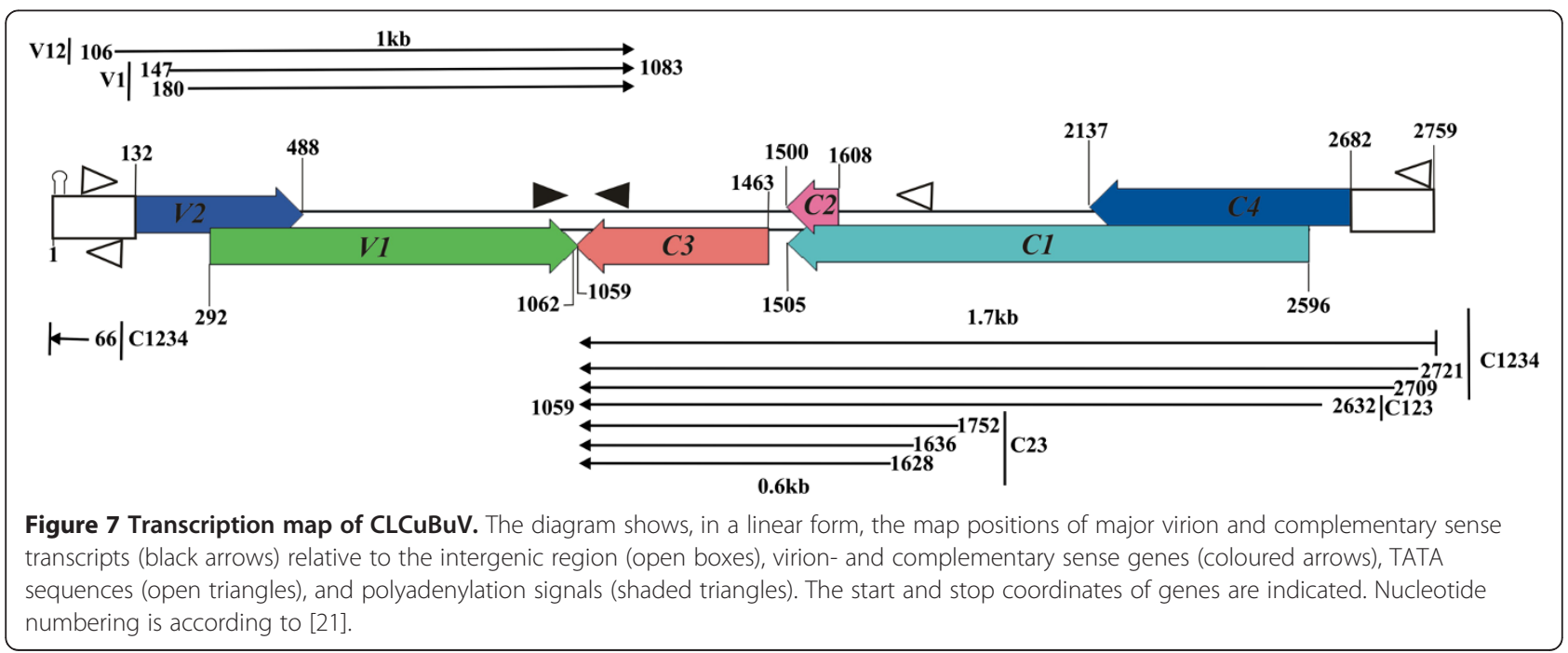


context within, and also on its distance from, the $5^{\prime}$ UTR [37]. Short $5^{\prime}$ UTRs may be associated with a decrease in translation initiation efficiency and vice versa [38].

The RACE clone sequences for the complementarysense showed a single predominant $3^{\prime}$ end located between the converging $V 1$ and $C 3$ genes. Transcription termination occurs immediately following the stop codon for the C3 gene. These transcripts do not have $3^{\prime}$ UTRs - polyadenylation occurs immediately following the stop codon of the $C 3$ gene. The poly (A) tail of the mRNA is added 21 nt downstream of canonical AATAAA polyadenylation signal. This site is located at an optimal distance downstream of a consensus polyadenylation termination signal suggesting that it represents the authentic $3^{\prime}$ end for these transcripts. Polyadenylation signals normally occur $10-30$ nt upstream of the polyadenalytion site [15]. The $3^{\prime}$ UTR for virion-sense transcripts determined in this study are short, being 21 nt. The poly (A) tail is added $22 \mathrm{nt}$ downstream of a polyadenylation signal. A similar arrangement has been observed for other geminiviruses; MYMV and SpCTV [29,31].

The single transcript identified for CLCuMuB maps with $5^{\prime}$ and $3^{\prime}$ ends at coordinates 563 and 190, respectively (Figure 6). These results are in agreement with the previous transcript mapping of the non-recombinant CLCuMuB [30]. The transcript is polyadenylated and the polyadenylation signal is $18 \mathrm{nt}$ upstream of the stop codon. The putative polyadenylation signal of the nonrecombinant CLCuMuB (AAATAA) differs from that of recombinant CLCuMuB (GAATAA). In contrast the putative TATA box element is the same for both CLCu$\mathrm{MuB}$ isolates, being $43 \mathrm{nt}$ upstream of the start codon and 31 nt upstream of transcription start site. These results support the predicted TATA box and start codon for $\beta C 1$ and suggest that transcription may be initiated from a consensus TATA box sequence at an optimal distance of 20-35 nt. The $5^{\prime}$ UTR identified here is of $12 \mathrm{nt}$ long and a transcript with a short leader sequence of eight nucleotides has previously been observed for AYVB [15]. The critical length of leader sequences is 7 nucleotides [39]. The differences between the results obtained here and those of previously obtained for AYVB [15] (multiple widely spaced 3' termini and some minor $5^{\prime}$ termini) and for the non-recombinant CLCuMuB (one additional minor $3^{\prime}$ and $5^{\prime}$ terminus) [30], may be due to differences in experimental approach. For both previous analyses of $\beta C 1$ transcripts, RNA was extracted from transgenic plants harbouring dimeric betasatellite constructs - such plants would not be expected to contain episomally replicating satellite. The transcripts here are from a bona fide begomovirusbetasatellite infection. However, Saunders et al. [15] attributed some of the diversity in $3^{\prime}$ termini to the presence of a cryptic polyadenylation in the sequence of AYVB. No such cryptic polyadenylation signals (although the sequence of the presumed polyadenylation signal differs from the consensus, as mentioned earlier) are present in the recombinant CLCuMuB.

Sequencing of clones of the short transcript spanning the $\mathrm{C} 2$ mutation in $\mathrm{CLCuBuV}$ did not show any splicing, or other editing event, which might restore expression of a full-length C2 protein, supporting the conclusion of Amrao et al. [21] that an intact $\mathrm{C} 2$ is not expressed by CLCuBuV. However, the limited number of clones analyzed here and the limitations of the RLM-RACE technique, namely that it cannot produce the sequences of full length transcripts, means that the results must remain tentative. It may be desirable to further analyse CLCuBuV transcription, using for example circularization reverse transcriptase PCR, to determine the full length sequences of viral transcripts and identify any possible low abundance transcripts. Moreover, northern blotting and quantitative RT-PCR would also be helpful to compare the expression of early and late genes at various stages of replication cycle.

The transcription of $\mathrm{CLCuBuV}$ and the recombinant $\mathrm{CLCuMuB}$ is equivalent to earlier begomoviruses/betasatellites that were transcription mapped. The recombinations/mutations that led to their appearance caused no detectable differences at the transcription level. Nevertheless, the study provides some avenues of investigation to follow-up in the efforts to determine the mechanism of resistance breaking in cotton by CLCu$\mathrm{BuV}$ and its betasatellite. These include the possible differences in the $\mathrm{C} 4$ protein, or expression thereof, and possible effects on REn expression, in addition to the hypothesis put forward by Amrao et al. [21] that the C2 protein may have been the avirulence determinant of the pre-resistance breaking virus species that was recognized by resistant cotton. It is also important to note that the CLCuBuV REn is chimeric, consisting of sequences derived from both CLCuMuV and CLCuKoV [21]. These possibilities will be the subject of future investigation of the mechanism of resistance breaking in cotton.

\section{Competing interests}

The authors declare that they have no competing interests.

\section{Authors' contributions}

FA performed the experiments and prepared the first draft of the manuscript. MS provided overall directions regarding the designing of all experiments, writing and supervised the work. FV supervised the hybridization experiments during the short-term visit of FA in Basel and discussed the different results with FA. RWB was involved in critical review of the work and in writing the manuscript. The final manuscript was read and approved by all authors.

\section{Acknowledgements}

FA was supported by the Higher Education Commission (HEC, Pakistan) under the 'Indigenous 5000 Fellowship Scheme'. RWB was supported by the 
HEC under the 'Foreign Faculty Hiring Scheme'. The work in the group of FV was funded by the HEC with a grant under the "International Research Support Initiative Programme" to FA.

\section{Author details}

'Agricultural Biotechnology Division, National Institute for Biotechnology and Genetic Engineering, Jhang Road, Faisalabad, Pakistan. ${ }^{2}$ Zürich-Basel Plant Science Center, Part of the Swiss Plant Science Web, Botanical Institute of the University of Basel, Schönbeinstraße $6 \mathrm{CH}-4056$, Basel, Switzerland.

Received: 6 June 2012 Accepted: 25 October 2012 Published: 29 October 2012

\section{References}

1. Brown JK, Fauquet CM, Briddon RW, Zerbini M, Moriones E, Navas-Castillo J: Geminiviridae. In Virus Taxonomy - Ninth Report of the International Committee on Taxonomy of Viruses. Edited by King AMQ, Adams MJ, Carstens EB, Lefkowitz EJ. London, Waltham, San Diego: Associated Press, Elsevier Inc; 2012:351-373.

2. Briddon RW, Stanley J: Sub-viral agents associated with plant singlestranded DNA viruses. Virology 2006, 344:198-210

3. Hanley-Bowdoin L, Settlage SB, Orozco BM, Nagar S, Robertson D: Geminviruses: models for plant DNA replication, transcription, and cell cycle regulation. Crit Rev Plant Sci 1999, 18:71-106.

4. Briddon RW, Pinner MS, Stanley J, Markham PG: Geminivirus coat protein replacement alters insect specificity. Virology 1990, 177:85-94

5. Rojas MR, Jiang H, Salati R, Xoconostle-Cázares B, Sudarshana MR, Lucas WJ, Gilbertson RL: Functional analysis of proteins involved in movement of the monopartite begomovirus, Tomato yellow leaf curl virus. Virology 2001, 291:110-125.

6. Amin I, Hussain K, Akbergenov R, Yadav JS, Qazi J, Mansoor S, Hohn T, Fauquet CM, Briddon RW: Suppressors of RNA silencing encoded by the components of the cotton leaf curl begomovirus-betasatellite complex. Mol Plant Microbe Interact 2011, 24:973-983.

7. Glick E, Zrachya A, Levy Y, Mett A, Gidoni D, Belausov E, Citovsky V, Gafni Y: Interaction with host SGS3 is required for suppression of RNA silencing by tomato yellow leaf curl virus V2 protein. Proc Natl Acad Sci USA 2008, 105:157-161.

8. Sunter G, Bisaro DM: Transactivation of geminivirus AR1 and BR1 gene expression by the viral AL2 gene product occurs at the level of transcription. Plant Cell 1992, 4:1321-1331.

9. Dry I, Krake L, Mullineaux P, Rezaian A: Regulation of tomato leaf curl viral gene expression in host tissues. Mol Plant Microbe Interact 2000, 13:529-537.

10. Hohn T, Vazquez F: RNA silencing pathways of plants: Silencing and its suppression by plant DNA viruses. Biochim Biophys Acta 2011, 1809:588-600.

11. Settlage SB, See RG, Hanley-Bowdoin L: Geminivirus C3 protein: replication enhancement and protein interactions. J Virol 2005, 79:9885-9895.

12. Saunders K, Bedford ID, Briddon RW, Markham PG, Wong SM, Stanley J: A unique virus complex causes Ageratum yellow vein disease. Proc Natl Acad Sci USA 2000, 97:6890-6895.

13. Briddon RW, Mansoor S, Bedford ID, Pinner MS, Saunders K, Stanley J, Zafar Y, Malik KA, Markham PG: Identification of DNA components required for induction of cotton leaf curl disease. Virology 2001, 285:234-243.

14. Jose J, Usha R: Bhendi yellow vein mosaic disease in India is caused by association of a DNA $\beta$ satellite with a begomovirus. Virology 2003, 305:310-317.

15. Saunders K, Norman A, Gucciardo S, Stanley J: The DNA $\beta$ satellite component associated with ageratum yellow vein disease encodes an essential pathogenicity protein (BC1). Virology 2004, 324:37-47.

16. Qazi J, Amin I, Mansoor S, lqbal J, Briddon RW: Contribution of the satellite encoded gene $\beta C 1$ to cotton leaf curl disease symptoms. Virus Res 2007 128:135-139.

17. Saeed M, Zafar Y, Randles JW, Rezaian MA: A monopartite begomovirusassociated DNA $\beta$ satellite substitutes for the DNA B of a bipartite begomovirus to permit systemic infection. J Gen Virol 2007, 88:2881-2889.

18. Mansoor S, Amin I, Briddon RW: Geminiviral diseases of cotton. In Stress Physiology in Cotton. Volume 7. Edited by Oosterhuis DM. Tennessee, U.S.A: The Cotton Foundation, Cordova; 2011.

19. Mansoor S, Briddon RW, Bull SE, Bedford ID, Bashir A, Hussain M, Saeed M, Zafar MY, Malik KA, Fauquet C, Markham PG: Cotton leaf curl disease is associated with multiple monopartite begomoviruses supported by single DNA $\beta$. Arch Virol 2003, 148:1969-1986.

20. Mansoor S, Amin I, Iram S, Hussain M, Zafar Y, Malik KA, Briddon RW: Breakdown of resistance in cotton to cotton leaf curl disease in Pakistan. Plant Pathol 2003, 52:784

21. Amrao L, Amin I, Shahid S, Briddon RW, Mansoor S: Cotton leaf curl disease in resistant cotton is associated with a single begomovirus that lacks an intact transcriptional activator protein. Virus Res 2010, 152:153-163.

22. Zaffalon V, Mukherjee S, Reddy V, Thompson J, Tepfer M: A survey of geminiviruses and associated satellite DNAs in the cotton-growing areas of northwestern India. Arch Virol 2011, 157:483-495.

23. Rajagopalan PA, Naik A, Katturi P, Kurulekar M, Kankanallul RS, Anandalakshmi $R$ : Dominance of resistance-breaking cotton leaf curl Burewala virus (CLCuBuV) in northwestern India. Arch Virol 2012, 157:855-868.

24. Amin I, Mansoor S, Amrao L, Hussain M, Irum S, Zafar Y, Bull SE, Briddon RW: Mobilisation into cotton and spread of a recombinant cotton leaf curl disease satellite. Arch Virol 2006, 151:2055-2065.

25. Townsend R, Stanley J, Curson SJ, Short MN: Major polyadenylated transcripts of cassava latent virus and location of the gene encoding coat protein. EMBO J 1985, 4:33-37.

26. Sunter G, Gardiner WE, Bisaro DM: Identification of tomato golden mosaic virus-specific RNAs in infected plants. Virology 1989, 170:243-250.

27. Frischmuth $\mathrm{S}$, Frischmuth $\mathrm{T}$, Jeske $\mathrm{H}$ : Transcript mapping of abutilon mosaic virus, a geminivirus. Virology 1991, 11815:596-604

28. Mullineaux PM, Rigden JE, Dry IB, Krake LR, Rezaian MA: Mapping of the polycistronic RNAs of tomato leaf curl geminivirus. Virology 1993, 193:414-423.

29. Shivaprasad PV, Akbergenov R, Trinks D, Rajeswaran R, Veluthambi K, Hohn $T$, Pooggin MM: Promoters, transcripts, and regulatory proteins of Mungbean yellow mosaic geminivirus. J Virol 2005, 79:8149-8163.

30. Saeed M, Behjatnia SAA, Mansoor S, Zafar Y, Hasnain S, Rezaian MA: A single complementary-sense transcript of a geminiviral DNA $\beta$ satellite is determinant of pathogenicity. Mol Plant Microbe Interact 2005, 18:7-14.

31. Baliji S, Sunter J, Sunter G: Transcriptional analysis of complementarysense genes in Spinach curly top virus and functional role of C2 in pathogenesis. Mol Plant Microbe Interact 2007, 20:194-206.

32. Akbergenov R, Si-Ammour A, Blevins T, Amin I, Kutter C, Vanderschuren $\mathrm{H}$ Zhang P, Gruissem W, Meins F Jr, Hohn T, Pooggin MM: Molecular characterization of geminivirus-derived small RNAs in different plant species. Nucleic Acids Res 2006, 34:462-471.

33. Gale M, Tan S-L, Katze MG: Translational control of viral gene expression in eukaryotes. Microbiol Mol Biol Rev 2000, 64:239-280.

34. Shung C-Y, Sunter G: Regulation of Tomato golden mosaic virus AL2 and AL3 gene expression by a conserved upstream open reading frame. Virology 2009, 383:310-318

35. Joshi $C P$, Zhou $H$, Huang $X$, Chiang VL: Context sequences of translation initiation codon in plants. Plant Mol Biol 1997, 35:993-1001.

36. Shung C-Y, Sunter J, Sirasanagandla SS, Sunter G: Distinct viral sequence elements are necessary for expression of Tomato golden mosaic virus complementary sense transcripts that direct AL2 and AL3 gene expression. Mol Plant Microbe Interact 2006, 19:1394-1405.

37. Kozak M: An analysis of $5^{\prime}$-noncoding sequences from 699 vertebrate messenger RNAs. Nucleic Acids Res 1987, 15:8125-8148.

38. Sedman SA, Gelembiuk GW, Mertz JE: Translation initiation at a downstream AUG occurs with increased efficiency when the upstream AUG is located very close to the $5^{\prime}$ cap. J Virol 1990, 64:453-457.

39. Kozak M: Effects of long $5^{\prime}$ leader sequences on initiation by eukaryotic ribosomes in vitro. Gene Expr 1991, 1:117-125.

\section{doi:10.1186/1743-422X-9-249}

Cite this article as: Akbar et al:: Transcript mapping of Cotton leaf curl Burewala virus and its cognate betasatellite, Cotton leaf curl Multan betasatellite. Virology Journal 2012 9:249. 